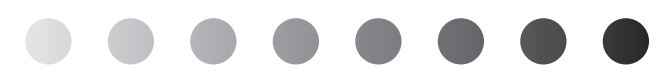

\title{
MEMORIA, IDENTIDAD Y SILENCIO: REFLEXIONES EN TORNO A LA NEGACIÓN DE ATROCIDADES DE LA INSURGENCIA SALVADOREÑA DURANTE LA GUERRA CIVIL
}

\author{
Jorge Juárez Ávila*
}

Resumen: En el presente artículo se reflexiona sobre el tratamiento de testimonios como fuentes para la investigación histórica de atrocidades, tal como el caso de la purga interna en una de las organizaciones que formaron la guerrilla salvadoreña, especialmente en el Frente Paracentral entre 1986 y 1991. Los testimonios de supervivientes, quienes fueron acusados de ser infiltres o agentes del "enemigo", nos trasladan a la interrelación entre memoria, identidad, silencio y negación de estas atrocidades. Además, se analiza cómo estas experiencias extremas de exmilitantes de la guerrilla están fuera de la memoria del FMLN, del Estado y la sociedad civil; en consecuencia, están cubiertas bajo toda una estrategia de negación.

Palabras clave: testimonios; memoria colectiva; guerra civil; negación, crimen de guerra; movimiento revolucionario; izquierda; Frente Farabundo Martí para la Liberación Nacional (FMLN); historia; El Salvador.

Abstract: The present article reflects on the use of testimonies as sources for historical research of atrocities, such as the case of internal purge in one of

Fecha de recepción: 01/05/2017-Fecha de aceptación: 01/07/2017

*Máster en Historia, Maestría Académica en Historia, Universidad de Costa Rica (UCR). Investigador del Instituto de Estudios Históricos, Antropológicos y Arqueológicos (IEHAA), Universidad de El Salvador, El Salvador. Coordinador de la Unidad de Investigaciones sobre la Guerra Civil Salvadoreña (UIGCS). Correo electrónico: javila62@gmail.com 
the organizations that formed the Salvadoran guerrilla, especially in the Paracentral front between 1986 and 1991. The testimonies of survivors who were accused of being infiltrators or agents of the "enemy" move us to the interrelation between memory, identity, silence and denial of these atrocities. Furthermore, it analyzes how these extreme experiences of ex-guerrilla members are outside the memory of the FMLN, the State and civil society; consequently, they are covered under a whole denial strategy.

Keywords: Testimonies; Collective Memory; Civil War; Denial; War Crimes; Revolutionary Movements; Left; Farabundo Martí National Liberation Front (FMLN); History; El Salvador.

\section{Introducción}

El objetivo de este artículo es reflexionar acerca del tratamiento historiográfico de acontecimientos controversiales como las purgas internas en la guerrilla salvadoreña. Estas reflexiones parten de un hecho concreto: la purga interna que se desató entre 1986 y 1991 en el Frente Paracentral ${ }^{1}$ de las Fuerzas Populares de Liberación (FPL) Farabundo Martí, una de las organizaciones que formaron el Frente Farabundo Martí para la Liberación Nacional (FMLN) en El Salvador.

Para abordar esta temática compleja, partimos de algunos planteamientos teóricos y metodológicos de Michael Pollack, fundamentalmente sobre el tratamiento de los testimonios de personas que vivieron situaciones límite y cómo: memoria, identidad y silencio están íntimamente relacionadas en la vida de estas personas. Por otro lado, tomamos algunos planteamientos de Stanley Cohen para analizar la negación de estas atrocidades. Incorporamos también la noción de Reinhart Koselleck de memoria negativa la cual-según Koselleck - tiene un sentido ambivalente, pues o bien alude a lo negativo de la memoria, de tal manera que el contenido almacenado repugna, no es bienvenido y se considera despreciable o digno de rechazo, o bien lo negativo significa que la memoria se cierra al recuerdo y rehúsa reconocer lo negativo: es decir, reprime, hace que se eluda el pasado y que se aporte olvido. Ambos, el contenido generador de lo horroroso y la inhibición a llevarlo de la memoria al recuerdo, están estrechamente relacionados. Uno remite al otro y nos llevan inmediatamente a la problemática historiográfica de cómo es posible el recuerdo de los crímenes.

1 El Frente Paracentral estaba constituido por los actuales Departamentos: San Vicente, Zacatecoluca y Cabañas. Hacemos alusión a este Frente en tanto fue allí donde se concentró la mayor cantidad de asesinatos de militantes de las FPL. Según algunos informantes, esta purga abarcó otros frentes de guerra, incluso en el exterior de El Salvador. 
Memoria, identidad y silencio: reflexiones en torno a la negación de atrocidades de la insurgencia salvadoreña durante la guerra civil

El artículo consta de cuatro partes. En primer lugar, hacemos una breve descripción de lo acontecido en el Frente Paracentral de las FPL entre 1986 y 1991. En segundo lugar, se plantea el dilema del autor de este artículo, quien fue militante de las FPL y ahora intenta comprender la lógica de estos acontecimientos desde la disciplina de la historia. En tercer lugar, hacemos un acercamiento a la importancia de los testimonios para el abordaje académico de estos acontecimientos. Finalizamos con las principales formas de negación de estas atrocidades.

\section{¿Qué pasó en el Frente Paracentral entre 1986 y $1991 ?$}

A partir del inicio de 1986, en el Frente Paracentral se configuró un estado generalizado de sospechas, intrigas y asesinatos de jefes y combatientes de las FPL acusados de ser infiltres o agentes del "enemigo". Ralph Sprenkels ha planteado que "... sin duda es una de las páginas más oscuras de la historia de la guerrilla, como un caso de purgas internas de supuestos enemigos infiltrados y otros agentes contrarrevolucionarios. Desde luego no fue el único, pero fue con mucho, el caso más extremo...". ${ }^{2}$ Ciertamente es el caso más extremo de una práctica de violencia política al interior de las organizaciones político-militares, que se puede rastrear desde los inicios de su fundación, basta con recordar el asesinato del poeta Roque Dalton dentro de las filas del ERP o los sucesos al interior de las FPL con el asesinato de Mélida Anaya Montes y luego el "suicidio" de Salvador Cayetano Carpio, segunda y primer responsables de las FPL en abril de 1983. Hasta hoy no sabemos el número de asesinatos cometidos por la FPL en el Frente Paracentral, algunos supervivientes y exmilitantes hablan de cifras cercanas al millar. El comandante que dirigió esta purga interna fue Mayo Sibrián, un militante de las FPL que gozaba de toda la confianza de la dirección de dicha organización político militar. Hasta hoy no tenemos un perfil exhaustivo de Mayo Sibrián, -su personalidad, su

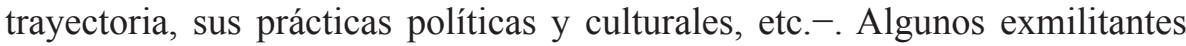
que lo conocieron se refieren a él como un hombre duro, de carácter fuerte, fogueado en lo cruento de los combates militares y que había sobrevivido a la experiencia de ser prisionero del "enemigo".

2 Ralph Sprenkels, Revolution and Accommodation. Post-insurgency in El Salvador (Universidad de Utrecht, s. f.), 308. 
Estuvo preso en las bartolinas de la fuerza armada en 1984 y fue liberado en octubre de $1985^{3}$. Luego de su experiencia en las cárceles del régimen autoritario salvadoreño, las FPL lo enviaron al Frente Paracentral para hacerse cargo de su conducción como máximo jefe político militar. Tampoco contamos con una reconstrucción del escenario de la guerra en esta zona en el que se incluya las estrategias de los dos bandos principales: El FMLN y las fuerzas armadas apoyadas por los Estados Unidos. En este escenario deben ser prioritarios los temas de "información e inteligencia", así como los niveles de apoyos de ambos bandos en contienda, ya que en estos procesos de guerras civiles los combatientes irregulares y los espías y agentes de cualquiera de las dos partes se esconden entre la población civil. ${ }^{4}$ Probablemente, este abordaje pendiente nos ayudará en un futuro a comprender mejor este acontecimiento. Por el momento, nos interesa comprender la gestión de la memoria: silencios, olvidos y negaciones de estos acontecimientos traumáticos analizados a partir de los testimonios de algunos supervivientes de estas purgas.

Con esta breve descripción se pretende exponer una idea general sobre un acontecimiento traumático de gran alcance, que cargó con un peso extraordinario a muchos militantes guerrilleros que tuvieron que convivir no solo con el horror de una guerra, que ya es mucho, sino con una situación límite en la que fueron acusados de "traición" y posteriormente asesinados. Muchos murieron y nunca obtendremos su testimonio ya que son experiencias intransferibles. Por suerte, ahora contamos con algunos testimonios de supervivientes y poco a poco vamos abriendo paso para comprender la lógica de esos crímenes. Por el momento, podemos afirmar con toda rigurosidad que esta purga interna - probablemente la más grande dentro de una organización de izquierda en América latina- sí existió.

La bibliografía que aborda este acontecimiento es limitada. En realidad no hay una investigación académica. No obstante, hay algunas iniciativas en las que sin ser el tema central se hace mención. ${ }^{5}$ Ralph Sprenkels, en su tesis doctoral, también hace referencia a esta temática. Y dejo en último lugar, el

3 Oscar Martínez Peñate, "Mayo Sibrián: las locuras de un comandante del FMLN (relato)", Realidad y Reflexión (El Salvador) 21 (septiembre-diciembre 2007): 9-19, disponible en URL: https://www.researchgate.net/publication/316887860_Mayo_Sibrian_las_locuras_de_ un_Comandante_del_FMLN_relato.

4 Stathis Kalyvas, La lógica de la violencia en la guerra civil (Madrid, España: Ediciones Akal, 2010), 133.

$5 \mathrm{Al}$ respecto podemos citar a: Tom Gibb, Under the Shadow of dreams. El Salvador's Revolutionaries (Manuscrito, 2000); Vincent McElhinny, Inequality and Empowerment: the Political Foundation of Post-War Decentralization and Development in El Salvador (1992-2000) (Tesis doctoral, University of Pittsburg, 2006); Iván Castro, "Niños perdidos en la guerra. La historia de Emiliano", Tendencias, 75 (1999): 11-1. 
Memoria, identidad y silencio: reflexiones en torno a la negación de atrocidades de la insurgencia salvadoreña durante la guerra civil

libro Grandeza y miseria en una guerrilla: informe de una matanza, publicado por Geovani Galeas y Berne Ayalá en $2008,{ }^{6}$ por considerarlo algo así como un arma de doble filo, ya que por un lado pone ante la opinión pública un tema tan delicado, basado en una gran cantidad de informantes, pero que al mismo tiempo fue muy criticado por el momento en el que fue presentado, es decir en medio de la campaña electoral en la cual Sánchez Cerén iba como candidato a la vicepresidencia junto a Mauricio Funes. Personalmente pienso que este tipo de libros están fuera del ámbito académico, ya que minimiza o deja de lado lo fundamental: entender la lógica de la violencia política al interior de las organizaciones guerrilleras, así como el tratamiento o la gestión de la memoria, los olvidos, el silencio y la negación por la sociedad, las familias de las víctimas, los supervivientes y el Estado. A pesar de eso, es un libro que debemos consultar por la riqueza de la información recopilada a través de un extenso trabajo de campo. Tomando como base esta bibliografía limitada, es que planteamos algunas ideas a partir de nuestra propia experiencia.

\section{Mi propia experiencia: entre memoria e historia}

Cuando decidí tomar este tema de investigación, el primer dilema que experimenté fue: por un lado, haber sido militante de las FPL desde 1979 hasta el final de la guerra civil y haber estado en el Frente Paracentral de 1985 a 1987 y de 1989 a 1990. Por lo tanto poseo una memoria individual y una memoria común que es la de una generación joven de izquierda de aquella época -con todas las implicaciones políticas, ideológicas y traumáticas que conlleva-. Por otro lado, ahora, veinticinco años después de finalizada la guerra, intento comprender la lógica de esos acontecimientos desde mi posición de historiador. Entonces, ¿cuál es el dilema? Como lo ha planteado Michael Pollack, es un proceso que consiste en estudiar "científicamente", es decir fríamente y a distancia, cosas que suscitan las reacciones afectivas más extremas y que son de ordinario abordadas en el registro "caliente" de la revuelta, de la denuncia o de la indignación. Por su carácter extremo, un objeto así pone en evidencia lo característico de todo emprendimiento científico, es decir, para emplear una imagen, producir frío allí donde sopla lo caliente o aún, para retomar el término de Norbert Elías, imponer el distanciamiento allí donde el objeto de estudio llama espontáneamente a una extrema "implicación". ${ }^{7}$ En todo caso, para que este tipo de trabajos de investigación surjan no solo es necesario que haya

6 Geovani Galeas y Berne Ayalá, Grandeza y miseria en una guerrilla: informe de una matan$z a$ (San Salvador, El Salvador: Centroamerica 21), 2008.

7 Michael Pollack, Memoria, olvido, silencio. La producción social de identidades frente a situaciones límite (La Plata, Argentina: Ediciones Al Margen, 2006), 58. 
alguien que vivió una situación extrema que quiera testimoniar, sino también alguien que quiera contarla o hacerla pública y esa sola decisión creo que es un buen punto de partida para el distanciamiento. Sobre todo alguien como yo que vivió de cerca aquellos acontecimientos.

\section{Los testimonios como fuentes de experiencias en situaciones límites: identidad y silencio}

El segundo elemento que quiero plantear está relacionado con las fuentes para el estudio de estos acontecimientos traumáticos. ¿Estamos ante hechos insondables? Para la historia se vuelve un problema. ¿Dónde están las huellas? Definitivamente la experiencia de esas mil u ochocientas o setecientas víctimas directas del Frente Paracentral no la tendremos nunca, son experiencias intransferibles. ${ }^{8}$ A pesar de tanto silencio, olvido y negación, a través del testimonio de algunos supervivientes podemos encender alguna luz sobre la memoria de estas víctimas directas, así como la memoria de sus familiares. En estos casos descubrimos el poder de los testimonios, con todos sus matices, limitaciones, etc., se vuelven de primer orden ante el cierre sistemático de las fuentes para un tratamiento histórico. Retomando la idea de Michael Pollack referente al uso de testimonios, es pertinente tomar en cuenta no solo la voluntad de hablar de los afectados -en este caso, los supervivientes del Paracentral y sus familiares-, sino, principalmente, sus dificultades para preservar los sentimientos de identidad. De allí - plantea Pollack- que memoria y testimonio colocan en juego "no solamente la memoria, sino también la reflexión sobre uno mismo". ${ }^{9}$ Los testimonios pasan a ser considerados como verdaderos instrumentos de reconstrucción de la identidad y no solamente relatos factuales, limitados a una función informativa. Por otro lado, la reflexión sobre estos testimonios nos reenvía al problema del silencio. ${ }^{10}$

Para Pollack, la memoria es un elemento constituyente del sentimiento de identidad, tanto individual como colectiva, en la medida en que es también un componente muy importante del sentimiento de continuidad y de coherencia de una persona o de un grupo en su reconstrucción de sí. En el caso que nos ocupa, se trata de la construcción individual y colectiva de una identidad compleja, en una organización guerrillera que se caracterizó por ser la más coherente en su intención de "diferenciarse de los otros". Por la extensión de este artículo no se puede ahondar en todos los elementos constitutivos de

8 Reinhart Koselleck, Modernidad, culto a la muerte y memoria nacional (Madrid, España: Centro de Estudios Políticos y Constitucionales, 2011), 55.

9 Pollack, Memoria, olvido, silencio..., 13.

$10 \mathrm{Ibid}$. 
Memoria, identidad y silencio: reflexiones en torno a la negación de atrocidades de la insurgencia salvadoreña durante la guerra civil

la identidad de los militantes de la FPL ${ }^{11}$ me limitaré a mencionar que hubo todo un proceso bien organizado, con niveles jerárquicos de compromisos, que fueron constituyendo un sentido de pertenencia e identidad de sus militantes -"hombres nuevos"- capaces de hacer los más grandes sacrificios por una "causa" que se sacralizó. O como lo plantea Ralph Sprenkels, las organizaciones político-militares manejaron, en lo fundamental, un esquema organizativo marxista leninista, y entre otros elementos hay que destacar la construcción de la organización como "partido de cuadros", un sistema que moldeaba cuidadosamente una vanguardia revolucionaria con base en la transformación personal, el espíritu de sacrificio y el servicio incondicional al partido y la internalización de la "mística" revolucionaria. ${ }^{12}$

Muchos de estos militantes se iniciaron siendo niños, por ejemplo: Pablo, un excombatiente que junto a su familia se incorporó a las FPL y es un superviviente de la purga del Paracentral, a quien le asesinaron a su novia y a toda su familia, acusados de ser infiltres del "enemigo", y que por el hecho de ser el novio fue capturado y torturado por sus mismos compañeros, acusado de colaborar con "el enemigo". Pablo recuerda que posterior a esa experiencia de la cual salió vivo, una primera reacción fue volverse hermético, sin deseos de hacer ningún comentario. "Uno se preguntaba [plantea Pablo], por qué se estaban dando esas cosas con uno cuando estaba entregado... pues a morirse por defender la causa y luego sentirse traicionado por la misma causa...es algo irracional que a uno lo desconcierta porque uno siente como que en ese momento uno es algo que ya no sirve... hubo exceso de errores de parte de la dirección para tratar estos casos...y te sometían a esas pruebas que yo las veo como pruebas salvajes... Eso en alguna medida lo desconcierta a uno y lo mete en un conflicto interno... yo trataba de demostrar mi inocencia por lo que me acusaban y me interrogaban de esa manera, o sea un interrogatorio bien torturador fuera de todo lo común". ${ }^{13}$

Como ya mencioné, Pablo se unió a la guerrilla junto a su familia, pero una vez iniciado lo cruento de la guerra, poco a poco se fue desvinculando de ellos. Es al final de la guerra que vuelven a unirse y aparece de nuevo el dilema de cómo gestionar ese pasado, esa experiencia extrema en la que estaba

11 Ralph Sprenkels plantea que durante los años setenta, la izquierda revolucionaria salvadoreña fue señalada, frecuentemente, de ser sectaria. Las organizaciones político-militares salvadoreñas funcionaban, en esencia, como sectas.

12 Ralph Sprenkels, "Relaciones urbano-rurales en la insurgencia salvadoreña", en: Historia y debates sobre el conflicto armado salvadoreño y sus secuelas, (coord.) Jorge Juárez Ávila (San Salvador, El Salvador: UIGCS-IEHAA-Universidad de El Salvador; Fundación Friedrich Ebert, 2014), 27.

13 Pablo, entrevista, 25 de febrero de 2016. 
en juego su propia identidad, así como la de su familia. Como lo manifiesta nuestro entrevistado: "Me volví hermético cuando me reuní con mi familia, casi no hablaba porque sentía que regresaba al mismo punto del trauma y eso me impedía hablar de ese tema. Fue con el tiempo que comencé a platicarlo con mi familia y en un primer momento no quería decirles que había sido por una situación de desconfianza del partido hacia su gente". ${ }^{14}$ Pablo cuidaba que su familia digiriera con calma su experiencia y trató de hacerles sentir a ellos que lo que había sucedido en el Frente Paracentral era un simple efecto de lo cruento de una guerra, es decir, que estaban en juego los años de construcción de identidades individuales y colectivas, las cuales se verían fuertemente puestas en duda si Pablo contaba abiertamente lo sucedido. La gravedad de los conflictos identitarios, que han podido provocar estas situaciones, es lo que, a menudo, impide a las víctimas dar cuenta de ella. El silencio deliberado, obstáculo para toda investigación tendiente a reconstruir la lógica de las adaptaciones sucesivas ante rupturas radicales en el desarrollo de una vida, es sin duda el indicador más sobresaliente del carácter doblemente límite de estas experiencias: en el límite de lo posible, y por lo tanto en el límite de lo decible. No pueden así hablar de modo creíble sino aquellos que lo han sufrido, mientras que el esfuerzo por olvidar o no evocar públicamente puede ser una condición para superar ese pasado. ${ }^{15} \mathrm{La}$ experiencia de Pablo nos remite a la idea que Walter Benjamín planteaba en un fragmento de El narrador: “¿No se notó acaso que la gente volvía enmudecida del campo de batalla? En lugar de retornar más ricos en experiencias comunicables, volvían empobrecidos. Todo aquello que diez años más tarde se vertió en una marea de libros de guerra, nada tenía que ver con experiencias que se transmiten de boca a boca". ${ }^{16}$ También resulta interesante agregar un elemento más que tomamos de Koselleck: en los primeros veinte años de posguerra hemos estado ante una época de represión del recuerdo, ${ }^{17}$ es decir, aunque muchos sabíamos de estas atrocidades, no han estado en la agenda del recuerdo.

Pollack plantea que cada uno de los testimonios es resultado del encuentro entre la disposición del superviviente o afectado a hablar y las posibilidades de ser escuchado. Entre aquel que está dispuesto a reconstruir su experiencia biográfica, aquellos que le solicitan hacerlo o están dispuestos

14 Ibid.

15 Pollack, Memoria, olvido, silencio..., 55.

16 Walter Benjamín, El narrador (Valparaíso, Chile: Metales Pesados, 2008), 2.

17 Koselleck, Modernidad, culto a la muerte..., 44. 
a interesarse por su historia, se establece una relación social que define los límites de lo que es efectivamente decible. ${ }^{18}$

En otra entrevista realizada a una de las hijas de Rodrigo, un exmilitante de las FPL que ahora reside en Suecia y que estuvo en el Frente Paracentral durante todo el período de Mayo Sibrián, resulta de interés cómo ellas gestionan este tipo de traumas. No solo el hecho de haber nacido en el seno de una pareja que ya era guerrillera cuando ellas nacieron y eso las obligó a vivir con su abuela en su etapa de formación, sino el hecho de que ahora saben que algo feo les pasó a sus padres en el Paracentral. Ahora saben eso por determinados momentos, muy casuales, en los que sus padres han hablado algo de su experiencia traumática. Por ejemplo: cuando Carmen fue a visitar a su padre a Suecia, quien -como ya lo mencioné- emigró a ese país luego de finalizada la guerra civil en El Salvador, luego de los saludos y pláticas sobre la familia, Carmen le dice a su padre que lleva una encomienda por encargo. Su padre un poco molesto le reclama: "Porque te hiciste cargo de traerla. ¡Esa dirección está a casi tres horas en vehículo!". ${ }^{19}$ No obstante su reclamo, al día siguiente emprenden el viaje. Un poco fatigados por las horas de viaje, por fin llegan a su destino: tocan la puerta del domicilio y aparece un hombre mayor, pequeño, delgado y demacrado. Inmediatamente el padre de Carmen da la vuelta y queda en un estado de perplejidad. Partieron de inmediato de regreso a su casa. Pasaron un par de horas para que Rodrigo dijera las primeras palabras sobre lo sucedido. Carmen le pregunta: “¿Y qué te pasó?". Rodrigo le contesta un poco tembloroso: “EEra Mayo Sibrián!”. Es increíble cómo después de varios años de esa experiencia el trauma está a flor de piel. Es de imaginar el dilema interno de Rodrigo que lo obliga a mantener en silencio su experiencia. Además, esa reacción pone en cuestión el dato sobre la propia ejecución de Mayo Sibrián después de un juicio sumario al interior de las FPL. Aunque esto es otro tema, vale mencionar que en otro momento circunstancial en el que Rodrigo había bebido casi una botella de whisky, le contó a Carmen que una noche antes del fusilamiento de Mayo Sibrián, lo pusieron a él a cuidarlo toda la noche. Tratando de contrastar la información, platiqué con un exmilitante que estuvo en el Frente Paracentral después del fusilamiento de Mayo Sibrián y plantea que aunque todo indica que Mayo fue fusilado, hay que dejar un espacio a la duda. ¡Yo diría, al mito!

18 Pollack, Memoria, olvido, silencio..., 56.

19 Carmen, entrevista, 31 de octubre de 2016. 
Helena, la madre de Carmen, quien también estuvo en el Frente Paracentral en los años de Mayo Sibrián se rehúsa aún hoy a hablar del tema. Como ya lo mencionamos, la reflexión sobre los testimonios de supervivientes de situaciones traumáticas como las del Frente Paracentral nos remite al problema del silencio. Michael Pollack plantea que lejos de depender de la sola voluntad o de la capacidad de los testigos potenciales para reconstruir su experiencia, todo testimonio se ancla también y sobre todo en las condiciones sociales que lo vuelven comunicable, condiciones que evolucionan con el tiempo y que varían de un país a otro. Pero esa misma posibilidad de tornar públicos sus recuerdos condiciona por su parte el trabajo realizado para superar las crisis de identidad que están en el origen de la necesidad, y de la dificultad de testimoniar. ${ }^{20}$

\section{La negación de atrocidades: los muertos del Frente Paracentral}

Como lo ha planteado Stanley Cohen, en momentos de guerra y posguerra, sociedades enteras entran en procesos de negación masiva con consecuencias terribles, especialmente para las víctimas y los supervivientes, quienes se encuentran literalmente dislocados del tiempo histórico. ${ }^{21}$ En El Salvador, hay miles de personas que experimentaron atrocidades durante la guerra civil. Los Escuadrones de la Muerte y el propio ejército, cometieron innumerables crímenes. Algunas personas hablan de lo sucedido, pero todas en algún momento hemos entrado en algún nivel de negación. Del amplio y exhaustivo abordaje que Stanley Cohen hace de los estados de negación y su relación con el reconocimiento de las atrocidades y el sufrimiento humano en contextos políticos complejos, retomo nada más que algunas de las dimensiones del proceso de negación que involucra lo que Cohen denomina, el triángulo de la atrocidad, compuesto por las víctimas, contra quienes se actúa; los perpetradores, quienes cometen las atrocidades; y los observadores o espectadores, quienes ven y saben lo que está pasando.

En el caso de las víctimas del Frente Paracentral, no hay un reconocimiento de la autoría de esos asesinatos. Hay todo un esquema de negación que va desde la negación literal a una negación interpretativa, tal como las denomina Cohen. Por lo menos públicamente, podríamos considerar que estamos ante la primera denominación, o sea una negación literal, es decir una negación fáctica, el hecho o el conocimiento del hecho se desmiente o simplemente nunca se habla, aunque saben que están siendo cuestionados. Según alguna información proporcionada por un entrevistado, en el primer congreso de las

20 Pollack, Memoria, olvido, silencio..., 56.

21 Stanley Cohen, States of Denial: Knowing about Atrocities and Suffering (Nueva Jersey, EE. UU.: John Wiley \& Sons, 2013), 242. 
Memoria, identidad y silencio: reflexiones en torno a la negación de atrocidades de la insurgencia salvadoreña durante la guerra civil

FPL, posterior al final de la guerra, hubo un grupo de militantes que cuestionó fuertemente a la dirección por esos actos. Pero también, desde allí inició una suerte de negación interpretativa, es decir los hechos no se niegan, pero se les otorga un significado distinto al que es aparente. En ese caso no se niega lo que pasó, sino que se le da otro nombre o se reclasifican los hechos bajo una categoría distinta. Por ejemplo: un alto comandante de las FPL que dirigía junto a Mayo Sibrián el Frente Paracentral, se niega a hablar con profundidad sobre el tema y reconoce que hubo "errores", pero que "estábamos en guerra", que "aquello no era fácil". Muchos exmilitantes de las FPL, así como de otras organizaciones guerrilleras, incluso se niegan a hablar de "asesinatos" y aún hoy lo toman en la categoría de ajusticiamientos.

Otro informante que perdió a tres de sus hijos acusados de ser infiltres en el Paracentral nos proporcionó una extensa entrevista y pudimos platicar sobre sus hijos después de casi tres horas de entrevista, siempre redundaba en otra información, pero hablar de sus hijos se volvía incómodo. En la misma localidad donde él residía, a escasas tres cuadras vivía un reconocido perpetrador del Paracentral. Todos sabían que él había sido uno de los ejecutores de guerrilleros, pero todo se manejaba con suma cautela. Pudimos hacer una estropeada entrevista con el perpetrador, que se encontraba en un estado de salud sumamente precario y por supuesto no quiso hablar nada sobre su etapa en la guerrilla y en concreto de su etapa como hombre de confianza de Mayo Sibrián. En esa dimensión local los estados de negación varían de uno a otro pues conviven víctimas, perpetradores y observadores al mismo tiempo. Todos saben que algo feo pasó, pero la presión social acerca de la duda del reconocimiento de los muertos como víctimas de una atrocidad tremenda impide un tratamiento adecuado y devolverles la dignidad a esos muertos que ahora son traidores para unos y víctimas para otros.

En la actualidad los salvadoreños nos hemos vuelto observadores permanentes. Hemos visto como de un estado generalizado de guerra civil, con todas las implicaciones que esto tiene, hemos pasado a un estado generalizado de violencia social que de alguna manera ha impedido que muchas familias siquiera hayan tenido el tiempo de reflexionar. Por ejemplo: un informante que ahora vive en la región paracentral perdió varios familiares durante la guerra civil, pero en al momento que nos concedió una entrevista, hacía un mes que le habían asesinado a un hijo y el otro lo tenía preso acusado de pertenecer a las pandillas. En el momento de la entrevista se desmoronó completamente.

Tomando la idea de Reinhart Koselleck sobre lo que él denomina la memoria negativa: es decir, que el contenido almacenado en la memoria repugna, no es bienvenido y se considera despreciable o digno de rechazo, o bien lo negativo significa que la memoria se cierra al recuerdo y rehúsa reconocer lo 
negativo, ${ }^{22}$ podríamos plantear la pregunta acerca de ¿quién debe ser recordado? Por lo que podemos observar en el tratamiento de la memoria por el FMLN, los muertos del Paracentral están excluidos del recuerdo, por lo tanto, podemos plantear que en el FMLN actual hay una jerarquización de la muerte. Shafik Hándal constituye el paradigma del revolucionario y en su figura se condensa la idea del héroe de la revolución y, según los discursos de los dirigentes actuales, él es "la luz que ilumina el camino al futuro en El Salvador". Es poca o casi nula la alusión a otras figuras como mandos medios y mucho menos combatientes de primera línea y mucho menos a los muertos del Frente Paracentral.

\section{Conclusión}

Realizar el trabajo de campo, entrevistando a varios supervivientes de las atrocidades cometidas en el Frente Paracentral de la guerrilla salvadoreña, nos ha permitido un mayor acercamiento a la relación entre memoria, olvido y silencio. Tal como lo plantea Andreas Huyssen, ya no podemos seguir oponiendo memoria y olvido como solemos hacerlo, admitiendo en el mejor de los casos que el olvido viene a ser la inevitable imperfección y deficiencia de la memoria misma. ¿Acaso toda memoria no depende inevitablemente tanto de la distancia como del olvido, los mismos factores que subvierten su estabilidad y confiabilidad tan deseadas y al mismo tiempo son esenciales a la vitalidad de la memoria misma? ¿Acaso no constituye el poder esencial de la memoria el que pueda ser discutida desde nuevas perspectivas, desde evidencias novedosas, incluso desde los espacios que ella misma había bloqueado? ${ }^{23} \mathrm{El}$ tema que tratamos en este artículo está fuera del debate público de la memoria en El Salvador, las experiencias límites de estos exmilitantes guerrilleros están fuera de los discursos memoriales del FMLN, del Estado y la sociedad civil, más bien están cubiertas bajo toda una estrategia de negación. Salvo algunas expresiones desde el uso político de la memoria, ${ }^{24}$ el tema pertenece al mundo individual e íntimo de la memoria de los afectados directa e indirectamente por esta atrocidad.

Sin pretender otra cosa más que entender la lógica de estas atrocidades, probablemente estemos contribuyendo, desde el espectro académico, a abordar ante la sociedad estos temas polémicos. Nuestro entrevistado Pablo admite que lo que le ha permitido reintegrarse a la sociedad de la posguerra en

22 Koselleck, Modernidad, culto a la muerte..., 53.

23 Andreas Huyssen, En busca del futuro perdido. Cultura y memoria en tiempos de globalización (Buenos Aires, Argentina: Fondo de Cultura Económica, 2001), 144.

24 Por ejemplo: el uso de esta atrocidad en las campañas políticas electorales. 
Memoria, identidad y silencio: reflexiones en torno a la negación de atrocidades de la insurgencia salvadoreña durante la guerra civil

El Salvador ha sido precisamente el distanciamiento, el olvido y el silencio. Es muy posible que él no recuerde cosas, o que en el presente las vea de otra forma, eso nos confirmaría que no existe una pretendida memoria pura, pero por eso no deja de tener toda la importancia para poder entender qué es lo que está en juego. En este caso, el tema de la identidad es clave para entender las estrategias memoriales de los supervivientes del Frente Paracentral.

No emitimos ningún juicio moral del acontecimiento, pues tal como lo plantea Koselleck para otras experiencias, ¿quién puede decir que estos asesinatos son justos? Insistir en la afirmación moral es una afirmación repetida que solo puede constatar que aquello fue injusto. Esta afirmación no es argumento que nos permita ganar algún conocimiento adicional. De ahí que la explicación moral se halle en una situación aporética, pues por una parte no añade conocimiento alguno, pero, por otra, es necesaria como contraste a la pregunta de ¿cómo pudo ocurrir? ${ }^{25}$

\section{A manera de cierre: un cuento sobre la negación}

Cerramos con un pequeño cuento de Julio Cortázar, el cual recoge en gran parte lo que se quiere decir en este artículo. Esta idea se retoma del investigador colombiano Michael Reed Hurtado, quien utilizó en un artículo este mismo cuento para dejar abierta la reflexión acerca de los riesgos de no encarar la atrocidad.

Un grupo de argentinos decide fundar una ciudad en una llanura propicia, sin darse cuenta en su gran mayoría de que la tierra sobre la cual empiezan a levantar sus casas es un cementerio del cual no queda ninguna huella visible. Solo los jefes lo saben y lo callan, porque el lugar facilita sus proyectos, ya que es una planicie alisada por la muerte y el silencio y les ofrece la mejor infraestructura para trazar sus planos. Surgieron así los edificios y las calles, la vida se organiza y prospera, muy pronto la ciudad alcanza proporciones y alturas considerables y sus luces, que se ven desde muy lejos, son el símbolo orgulloso de quienes han alzado la nueva metrópolis. Es entonces cuando comienzan los síntomas de una extraña inquietud, las sospechas y los temores de quienes sienten que fuerzas extrañas los acosan y de alguna manera los denuncian y tratan de expulsarlos. Los más sensibles terminan por comprender que están viviendo sobre la muerte, y que los muertos saben volver a su manera y entrar en las casas, en los sueños, en la felicidad de los habitantes. Lo que parecía la realización de un ideal de nuestros tiempos, despierta lentamente a

25 Koselleck, Modernidad, culto a la muerte..., 46. 
la peor de las pesadillas, a la fría y viscosa presencia de repulsas invisibles, de una maldición que no se expresa con palabras pero que tiñe con su indecible horror todo lo que esos hombres levantaron sobre una necrópolis. ${ }^{26}$

26 Julio Cortázar, "Realidad y literatura en América Latina", Revista de Occidente, 5 (1981); 23-33. Tomado de: Michael Reed Hurtado, "Estados de negación: retos frente a la recuperación de la memoria en Colombia", en: Las víctimas. Entre memoria y olvido, (ed.) Ricardo García Duarte, Absalón Jiménez Becerra y Jaime Wilchez Tinjacá (Bogotá, Colombia: Universidad Distrital Francisco José de Caldas; Instituto para la Pedagogía, la Paz y el Conflicto Urbano; Centro de Memoria, Paz y Reconciliación; Secretaría de Gobierno de Bogotá, 2012). 